\title{
Organic Systems and Their Photorefractive Properties under the Nano- and Biostructuration: Scientific View and Sustainable Development
}

\author{
Natalia V. Kamanina, ${ }^{1,2}$ Sergey V. Serov, ${ }^{1}$ Yann Bretonniere, ${ }^{3}$ and Chantal Andraud ${ }^{3}$ \\ ${ }^{1}$ Lab for Photophysics of Media with Nanoobjects, Vavilov State Optical Institute, Kadetskaya Liniya V.O., Dom 5, \\ Korpus 2, St. Petersburg 199053, Russia \\ ${ }^{2}$ Saint-Petersburg Electrotechnical University "LETI", Prof. Popova Street, Dom 5, St. Petersburg 197376, Russia \\ ${ }^{3}$ Laboratoire de Chimie, UMR CNRS 5182, Ecole Normale Supérieure de Lyon, France
}

Correspondence should be addressed to Natalia V. Kamanina; nvkamanina@mail.ru

Received 30 October 2014; Accepted 19 December 2014

Academic Editor: Wei Hsuan Hung

Copyright (c) 2015 Natalia V. Kamanina et al. This is an open access article distributed under the Creative Commons Attribution License, which permits unrestricted use, distribution, and reproduction in any medium, provided the original work is properly cited.

Essential improvement of the photorefractive parameters of the organic structures has been found via nano- and biostructuring process. The spectral and self-assembling features which can support the photorefractive effect of some conjugated materials, including the liquid crystal ones, have been presented. To investigate the laser-induced change of the refractive index of the nanoand bio-objects-doped materials the holographic recording technique has been used. Moreover some innovative perspective dyes have been applied to study the effect of the rotation of the polarization plane of light. The area of application of the modified materials has been discussed. The tendency to replace the nanotechnology process with the biotechnology ones has been predicted.

\section{Introduction}

Extended area of the optoelectronic and biomedical application [1-5] of the different inorganic and organic compounds requires the search for new methods to optimize the spectral and photorefractive properties of such types of the materials. It is connected with an innovative design and simple way to control the basic physical-technical parameters.

According to the perspective role, namely, of the organic materials in optoelectronics and biomedicine in comparison with the inorganic ones, the optical features of these systems occupy the special place. Among these features the photorefractive parameters responsible for the effects of the laser-matter interaction in the anisotropic media have been considered in more detail.

In the current paper two optical effects coincided with the photorefractivity, such as the laser-induced refractive index changing, and the rotation of the plane of polarization of light is considered based on the model polyimide (PI) photosensitive donor-acceptor matrix and the nematic liquid crystal (NLC) compounds as well as on the solutions of noncentrosymmetric dyes. It should be mentioned that the organic systems with the initial intramolecular donoracceptor interaction have been used in order to have the chance to dope them with the intermolecular acceptors with good advantage.

\section{Theoretical Background}

In order to analyze the optical processes of the materials one should take into account the following. When the electric field of the laser wave is less than the intra-atomic electric field $E_{a}=e / a^{2}$ correlated with the electron charge $e$ and with the Bohr radius $a$, we should estimate the linear effect. But when the electric field of the laser wave is larger than the intra-atomic electric field $E_{a}$, we should draw the attention on the nonlinear optical features. Using this aspect, the values of optical susceptibility play important role in nonlinear optical effect. Really, the most important optical characteristic of all organic or inorganic materials with different symmetry 
is the induced dipole, which can be expressed through the dipole polarizabilities $\alpha^{(n)}$ [6]. These are in turn related by the proportional dependence to the nonlinear susceptibility $\chi^{(n)}$ and to the local volume $v$ of the materials (media). Thus, laser-matter interaction provokes the change in polarization of media and predicts the change in the important properties, such as dynamic, photorefractive, and photoconductive ones.

To predict the change of the cubic nonlinearity $\chi^{(3)}$, as minimum media local volume polarizability [7-9], the laserinduced change of the refractive index $\Delta n_{i}$ can be calculated from the diffraction efficiency $\eta[7-10]$ via realization of the Raman-Nath diffraction conditions $\left(\Lambda^{-1} \geq d\right)$ using

$$
\eta=\frac{I_{1}}{I_{0}}=\left(\frac{\pi \Delta n_{i} d}{2 \lambda}\right)^{2}
$$

Here $\Delta n_{i}$ is induced change of the refractive index, $I_{1}$ is the intensity in the first diffraction order, $I_{0}$ is the input laser intensity, $d$ is the thickness of the medium, $\lambda$ is the wavelength of the light incident on the medium, and $\Lambda$ is the spatial frequency. Due to the fact that the refractive index depends on the light intensity in the media with the cubic nonlinearity, the nonlinear refractive index $n_{2}$ can be estimated via (2) and then cubic nonlinearity $\chi^{(3)}$ can be found via (3):

$$
\begin{gathered}
n=n_{0}+n_{2} I, \\
n_{2}=\frac{16 \pi^{2}}{n_{0} c} \chi^{(3)} .
\end{gathered}
$$

Here $n_{0}$ is the linear refractive index and $c$ is the light velocity. It should be noticed that generally to calculate the nonlinear optical coefficients of the media with high value of the cubic nonlinearity we have used mechanism proposed in [7] and extended on the nanostructured materials in the publications [8-10].

Based on our previous results [8-12] and the current ones the model polyimide organic matrix and NLCs can be treated by pulsed nanosecond laser with good advantage to observe the dramatic change of the refractive index. Moreover, some anisotropic media based on the organic dyes solutions can support the evidences to use the innovative organic materials in the optical applications to resolve the different complicated tasks not only in the technical area but also in the biomedicine.

\section{Experimental Results and Discussion}

It should be remarked that the main accents are given on the study of the optical features of the structured organic materials.

\subsection{Photorefractive Features of the Organics Activated by} Laser Irradiation. The laser-induced change of the refractive index has been studied under the Raman-Nath diffraction conditions at spatial frequency of $90-120 \mathrm{~mm}^{-1}$ and at the laser energy density of $0.03-0.6 \mathrm{~J} \times \mathrm{cm}^{-2}$. The scheme has been analogous to that shown in [13] and can be shortly shown in Figure 1.

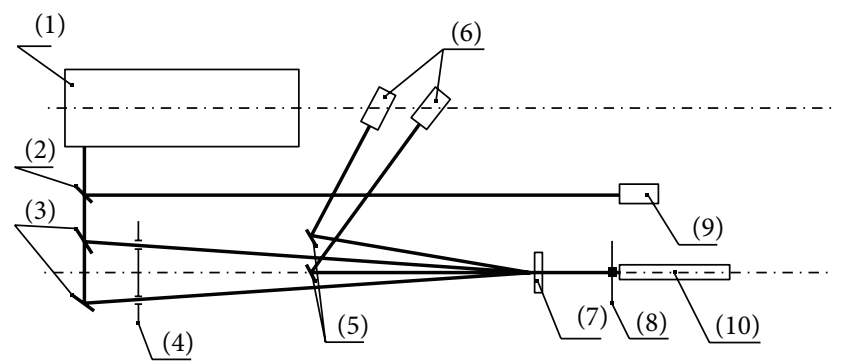

Figure 1: (1) Nd-laser with the converter to second harmonic, (2) plane-parallel plate, $(3,5)$ mirrors, $(4,8)$ blocking and absorbance diaphragms, (6) photodetectors, (7) samples, (9) synchronized device, and (10) an additional continuous Nd-laser to readout.

The wavelength of the second harmonic of the pulsed nanosecond Nd-laser was $532 \mathrm{~nm}$ and the laser pulse width was $10-30$ ns. The main results are shown in Table 1 . The database of the laser-induced change of the refraction index and thus general nonlinear features of the nano-objectsdoped materials with different sensitizers can be extended by including DNA.

Let us consider in detail results shown in Table 1. As the promising nanoobject fullerenes, the quantum dots, the shungites, and the carbon nanotubes have been considered as well as the bioparticles based on DNA, we have testified that the model consideration of domination of the intermolecular charge transfer under intramolecular one using different types and placement of the nanoobjects can be applied with good advantage. It should be mentioned that, in the sensitized organic conjugated system, for example, polyimide one, the pathway of the electrons included in the charge transfer process changes due to the fact that charge transfers from the intramolecular donor fragment of organic conjugated molecules (triphenylamine) not to its acceptor fragment (diimide) but to nanoobjects if the electron affinity energy of the nanoobjects is larger than the one for intramolecular acceptor fragment. For example, electron affinity energy of intramolecular acceptor fragment of polyimide is close to 1.1-1.4 eV, the electron affinity energy of shungite structure is $\sim 2 \mathrm{eV}$, the one for fullerenes is $\sim 2.65 \mathrm{eV}$, and the one for quantum dots is placed in the range of 3.8-4.2 eV. Thus, the intermolecular acceptor fragment electron affinity energy is essentially larger. Therefore, incorporated nanoobjects are stronger sensitizers and they dominate the acceptor fragments of intramolecular complexes. In this case the field gradient is formed, the additional dipole moment is observed, the increase of the local volume polarizability is found, and the increase of dipole polarizability and of the charge carrier mobility is revealed. The first consideration of this process has been made in [8] and extended in [9-12]. Regarding the DNA incorporation in the organic matrixes, it should be mentioned that these bioobjects revealed an additional shift in the spectral characteristics (that can support the possible formation of the charge transfer complex) which can be shown for the solution in Figure 2 and predicted the refractive index change with the value close to that observed before for the nano-object-doped organic materials. 
TABLE 1: Change of the refractive index $\Delta n_{i}$ of the doped polyimides and LCs.

\begin{tabular}{|c|c|c|c|c|}
\hline Numbers & Structure studied & Nanoobject content wt.\% & Energy density, $\mathrm{J} \times \mathrm{cm}^{-2}$ & $\Delta n_{i}$ \\
\hline 1 & Pure polyimide & 0 & 0.6 & $10^{-4}-10^{-5}$ \\
\hline 2 & Polyimide + malachite green dye & 0.2 & 0.6 & $2.87 \times 10^{-4}$ \\
\hline 3 & Polyimide + dye WD-C4 & 0.05 & 0.2 & $10.0 \times 10^{-3}$ \\
\hline 4 & Polyimide + QDs CdSe(ZnS) & 0.003 & $0.2-0.3$ & $2.0 \times 10^{-3}$ \\
\hline 5 & Polyimide $+\mathrm{C}_{70}$ & 0.2 & 0.6 & $4.68 \times 10^{-3}$ \\
\hline 6 & Polyimide $+\mathrm{C}_{60}$ & 0.2 & $0.5-0.6$ & $4.2 \times 10^{-3}$ \\
\hline 7 & Polyimide + shungite & 0.2 & $0.1-0.3$ & $5.3 \times 10^{-3}$ \\
\hline 8 & $\mathrm{NLC}+$ polyimide $+\mathrm{C}_{70}$ & 0.2 & $0.1-0.3$ & $1.2 \times 10^{-3}$ \\
\hline 9 & $\mathrm{NLC}+\mathrm{DNA}^{*}$ & 0.1 & 0.1 & $1.39 \times 10^{-3}$ \\
\hline 10 & $\mathrm{NLC}+\mathrm{QDs} \mathrm{CdSe}(\mathrm{ZnS})+\mathrm{DNA}$ & 0.1 & 0.1 & $1.35 \times 10^{-3}$ \\
\hline
\end{tabular}

${ }^{*}$ Content of DNA in the water solution was $\sim 4.72 \mathrm{~g} \times \mathrm{L}^{-1}$; the relation between LC and DNA was $5: 1$.

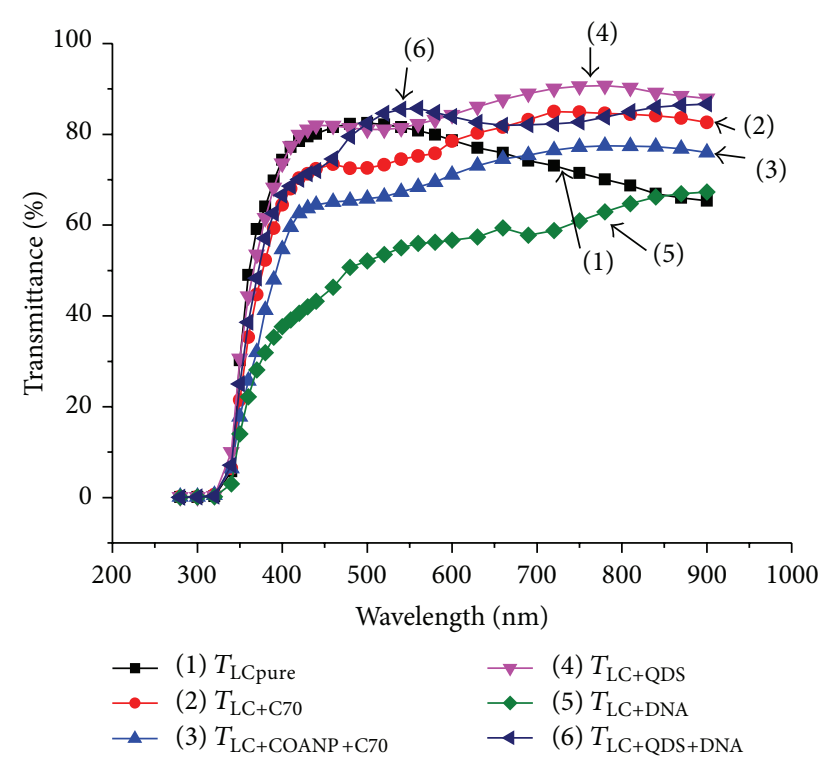

FIGURE 2: Spectral evidence of the formation of the CTC with DNA via observation of the bathochromic shift.

Indeed, the process of the DNA added in the organic matrix should be studied in detail in the future, but now one can testify that these renewable bioobjects can be considered as alternative ones to nanoobjects.

Using the obtained results, the nonlinear refraction $n_{2}$ and nonlinear third order optical susceptibility (cubic nonlinearity) $\chi^{(3)}$ for all systems have been calculated using a method described above. It was found that these parameters fall within the range: $n_{2}=10^{-10}-10^{-9} \mathrm{~cm}^{2} \times \mathrm{W}^{-1}$ and $\chi^{(3)}=$ $10^{-10}-10^{-9} \mathrm{~cm}^{3} \times \mathrm{erg}^{-1}$. It should be testified that the value of the obtained nonlinear optical coefficients for the organic materials studied in the current research is close to that received for the bulk silicon-based inorganic structures.

3.2. Rotation of the Plane of Polarization of Light. It should be mentioned that generally in order to operate with active media responsible for the rotation of the light polarization plane one can use classical $10 \%$ sugar-water solution. But, using the innovative dyes with less concentration in the solvent, we can observe this effect at the same experimental conditions. The experimental scheme to measure the angle of the polarization plane rotation is shown in Figure 3.

We have used the dyes as JM271-C1 (atomic weight is close to 317.4), YB-C2 (345.5), WD-C4 (401.6), JM47-C7 (485.7), and YB-C11 (597.9). Some dyes formulas are shown in Figure 4.

The spectral results regarding the dyes solution in the thetrachlorethan are shown in Figure 5(a). The change of the rotation angle for the solution with different dyes is shown in Figure 5(b).

One can see that all dyes solutions permit rotating the plane of polarization of light with good advantage. It should be mentioned once again that we have used less content of each dye in the thetrachlorethan solvent than ones applied for traditional 10\% sugar-water solution. Moreover, some saturation level has been observed for the molecular weight of dyes close to atomic weight of 375-400. It should be remarked that the $10 \%$ sugar-water solution rotates the light polarization plane with the angle of $3^{\circ}$ at the same thickness of the cuvette of $10 \mathrm{~mm}$. Thus these dyes can be considered as the alternative agents in comparison with sugar in order to realize the optical rotation effect for the polarization plane of light. Furthermore, for future comparative investigation, it will be necessary to functionalize the dyes in order to solve them in the water.

\section{Conclusion}

Based on the presented study and to analyze the received results one can say the following.

According to the database of the nonlinear features of the polyimide matrix and nematic liquid crystal materials from cyanobiphenyl group with different sensitizers, it can be prognosed that the different nanoobjects added in the model matrixes and new nature materials, for example, renewable DNA, can be considered as the good candidates to modify the photorefractive features of the organic materials with an initial donor-acceptor interaction. Moreover, it has been established that the nontoxic renewable bioparticles influence the photorefractive features of the organic conjugated 


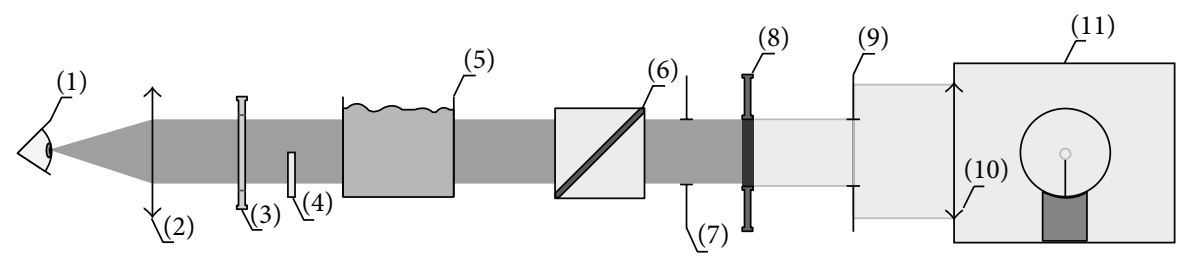

Figure 3: (1) Researcher's eye, (2) eyepiece, (3) polarizer, (4) rotated polarizes plate, (5) solution with dyes, (6) polarized prism, (7, 9) diaphragms, (8) filters, (10) lens, and (11) illuminator (lamp or He-Ne-laser at wavelength of $633 \mathrm{~nm}$ ).
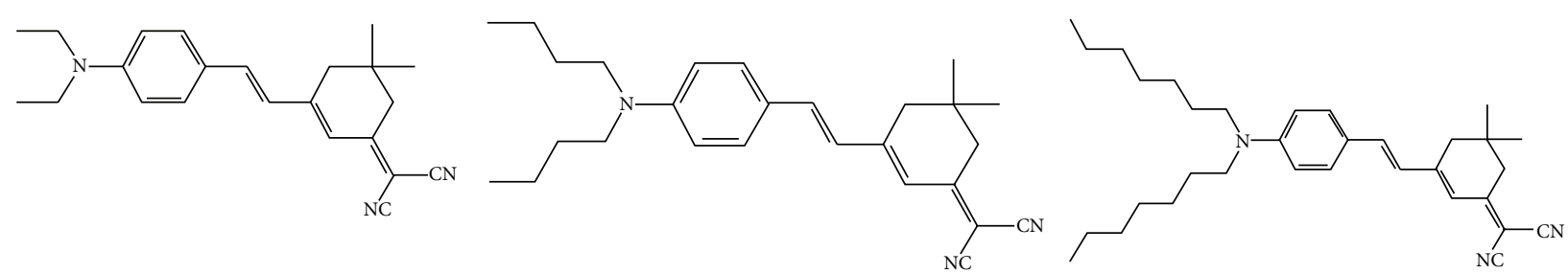

FIGURE 4: The structural formulas of some dyes used in the effect of the rotation of the polarization plane of the light: YB-C2, WD-C4, and JM47-C7 (from the left to right).
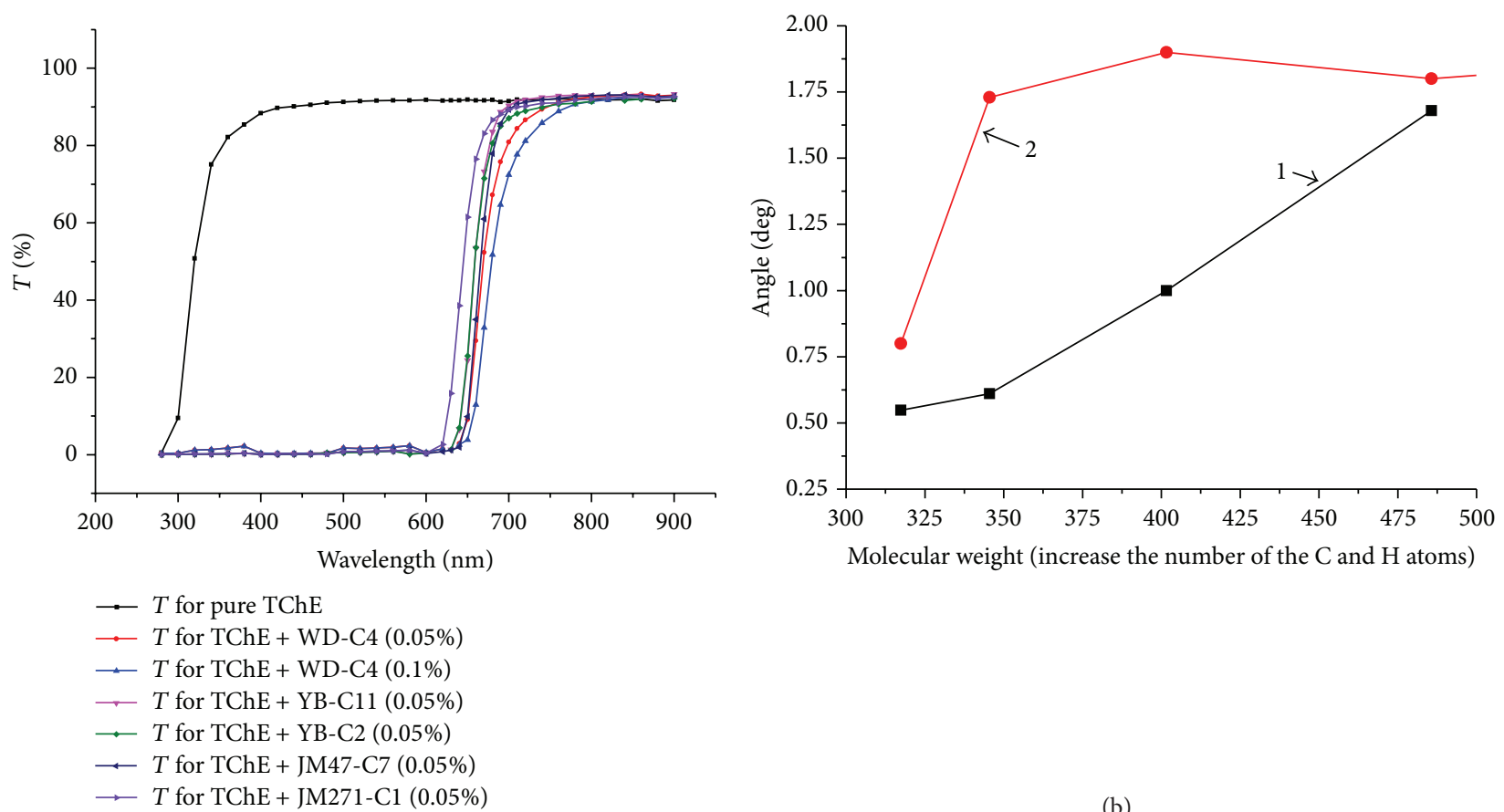

(a)

(b)

FIGURE 5: Transmittance spectra of the tetrachlorethane solutions of the dyes studied (a) and the dependence of the rotation angle of the polarization plane of light on the dyes molecular weight (b): (1) $0.05 \%$ solution and (2) $0.5 \%$ solution.

matrixes and can be predicted as alternative bioobjects instead of nanoobjects. For example, organic systems sensitized with the nanoparticles, such as fullerenes $\mathrm{C}_{60}$ and $\mathrm{C}_{70}$, carbon nanotubes, or quantum dots, can be replaced by the renewable bioparticles sensitized materials. It can predict to apply the organic materials with DNA for the holographic recording, optical limiting, and the testing of the dynamic properties of the DNA-modified composites and their possible application in the general telecommunication systems and in the biomedicine. Thus, as an important conclusion, we should tell and predict that the nanotechnology methods and components can be effectively replaced by the biotechnology ones.

Evidences to rotate the polarization plane of the light have been shown using innovative dyes in comparison with that for the general sugar-water solution. The threshold level for 
the rotation angle on molecular mass has been established. It has been mentioned that for the future experiments and their explanation the procedure to functionalize the dyes to solve them in water should be needed.

Some unique approaches proposed in this consideration possibly can be useful in order to collect the new knowledge in the nano- and biomaterials science and to extend the area of the application of the structured organic systems. The results presented can be useful to apply them in the education process because the nanomaterials study area permits visualizing some physical effects and explaining some laws of the laser-matter interaction under the conditions of nanodimensional scale. It should be noticed that this study can be useful in order to reveal the new knowledge in the nano- and biostructured organic materials science in comparison with that obtained for the classical inorganic crystals.

\section{Conflict of Interests}

The authors declare that there is no conflict of interests regarding the publication of this paper.

\section{Acknowledgments}

The authors would like to acknowledge their colleagues: Professor D. P. Uskokovic (Institute of Technical Sciences of the Serbian Academy of Sciences and Arts, Belgrade, Serbia), Brazilian Ph.D. student Rodrigo Sabadini, Professor F. Kajzar (Université d'Angers, Institut des Sciences et Technologies Moléculaires, France), and Dr. V. I. Studeonov and Dr. P. Ya. Vasilyev (Vavilov State Optical Institute, St. Petersburg, Russia) for their help at the different steps and helpful discussion. Some parts of the results presented in the current paper have been discussed at the scientific seminar, Czestochowa University (December 12, 2012, Poland), at ICBB'14 Conference, in Gdansk Polytechnic University (May 14-17, 2014, Poland), and at the Nanotechnology Session in the framework of the MMT-2014 Conference (July 28-August 1, 2014, Ariel, Israel). The presented results are partially supported by Russian Foundation for Basic Research Fund, Grant no. 13-03-00044 (2013-2015) as well as by the FP7 Program, Marie Curie International Researchers Exchange Proposal "BIOMOLEC" (2011-2015).

\section{References}

[1] K. Hosoda, R. Tada, M. Ishikawa, and K. Yoshino, "Effect of $\mathrm{C}_{60}$ doping on electrical and optical properties of poly[(disilanylene)-oligophenylenes]," Japanese Journal of Applied Physics, vol. 36, no. 3B, part 2, pp. L372-L375, 1997.

[2] W. Lee and H.-C. Chen, "Diffraction efficiency of a holographic grating in a liquid-crystal cell composed of asymmetrically patterned electrodes," Nanotechnology, vol. 14, no. 9, pp. 987990, 2003.

[3] I. C. Khoo, A. Diaz, D. Kwon et al., "Nonlinear and electrooptics of nano-dispersed nematic liquid crystals with tunable negative-, zero-, and positive indices," Journal of Nonlinear Optical Physics \& Materials, vol. 16, no. 3, pp. 381-399, 2007.
[4] A. D. Grishina, L. Licea-Jimenez, L. Y. Pereshivko et al., "Infrared photorefractive composites based on polyvinylcarbazole and carbon nanotubes," High Energy Chemistry, vol. 40, no. 5, pp. 341-347, 2006.

[5] Q. Peng, A. K. Dearden, J. Crean et al., "New materials graphyne, graphdiyne, graphone, and graphane: review of properties, synthesis, and application in nanotechnology," Nanotechnology, Science and Applications, vol. 7, no. 2, pp. 1-29, 2014.

[6] D. S. Chemla and J. Zyss, Eds., Nonlinear Optical Properties of Organic Molecules and Crystals, vol. 2, Academic Press, Orlando, Fla, USA, 1987, (Translated into Russian).

[7] S. A. Akhmanov and S. Y. Nikitin, Physical Optics, Oxford University Press, Oxford, UK, 1997.

[8] N. V. Kamanina, "Fullerene-dispersed nematic liquid crystal structures: dynamic characteristics and self-organization processes," Physics-Uspekhi, vol. 48, no. 4, pp. 419-427, 2005.

[9] N. V. Kamanina and D. P. Uskokovic, "Refractive index of organic systems doped with nano-objects," Materials and Manufacturing Processes, vol. 23, no. 6, pp. 552-556, 2008.

[10] N. V. Kamanina, A. Emandi, F. Kajzar, and A.-J. Attias, "Laserinduced change in the refractive index in the systems based on nanostructured polyimide: comparative study with other photosensitive structures," Molecular Crystals and Liquid Crystals, vol. 486, pp. 1-11, 2008.

[11] N. V. Kamanina, S. V. Serov, N. A. Shurpo et al., "Polyimidefullerene nanostructured materials for nonlinear optics and solar energy applications," Journal of Materials Science: Materials in Electronics, vol. 23, no. 8, pp. 1538-1542, 2012.

[12] N. V. Kamanina, Features of Optical Materials Modified with Effective Nanoobjects: Bulk Properties and Interface, Nova Science Publishers, Novinka, New York, NY, USA, 2014.

[13] N. V. Kamanina and N. A. Vasilenko, "Influence of operating conditions and interface properties on dynamic characteristics of liquid-crystal spatial light modulators," Optical and Quantum Electronics, vol. 29, no. 1, pp. 1-9, 1997. 

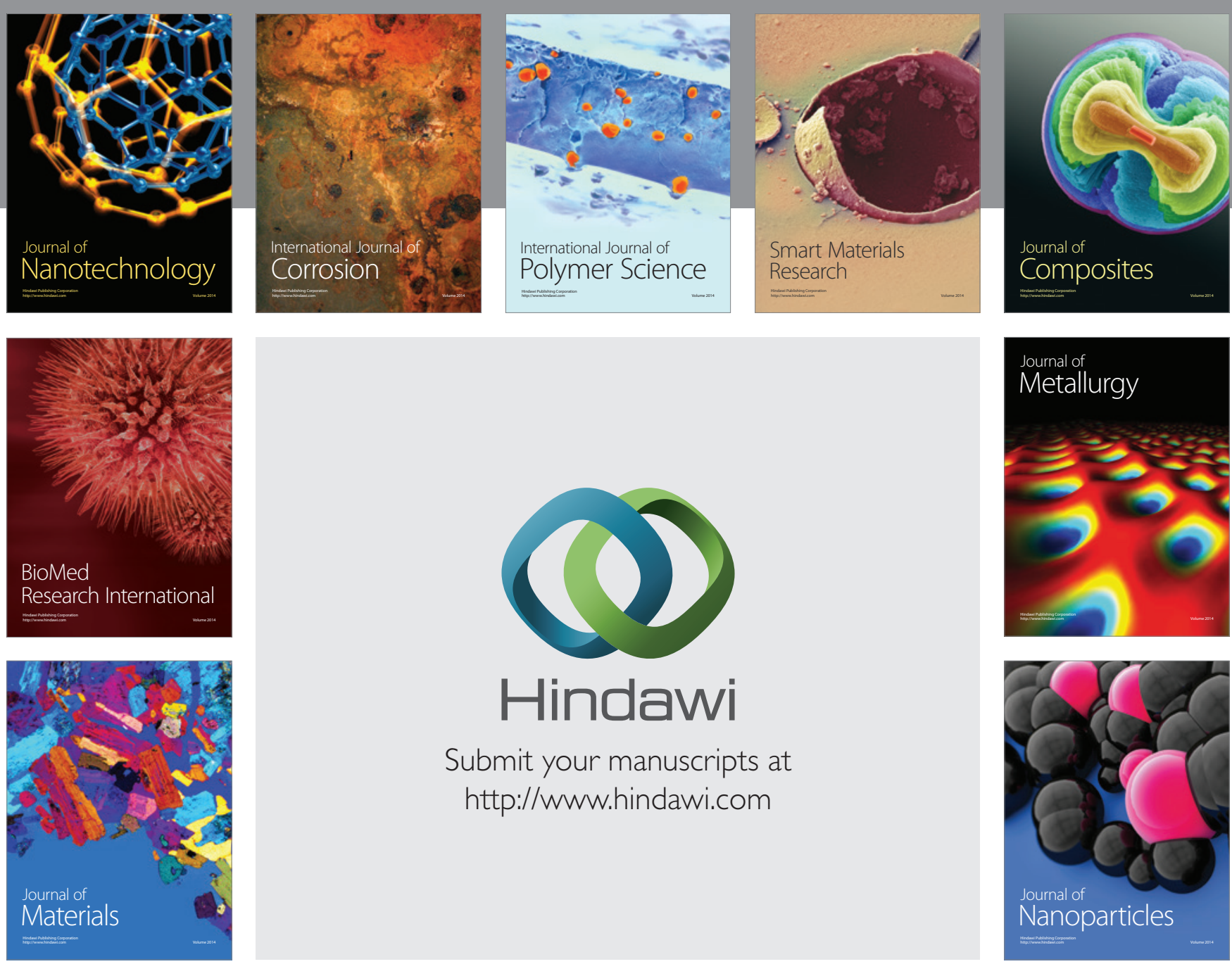

Submit your manuscripts at http://www.hindawi.com
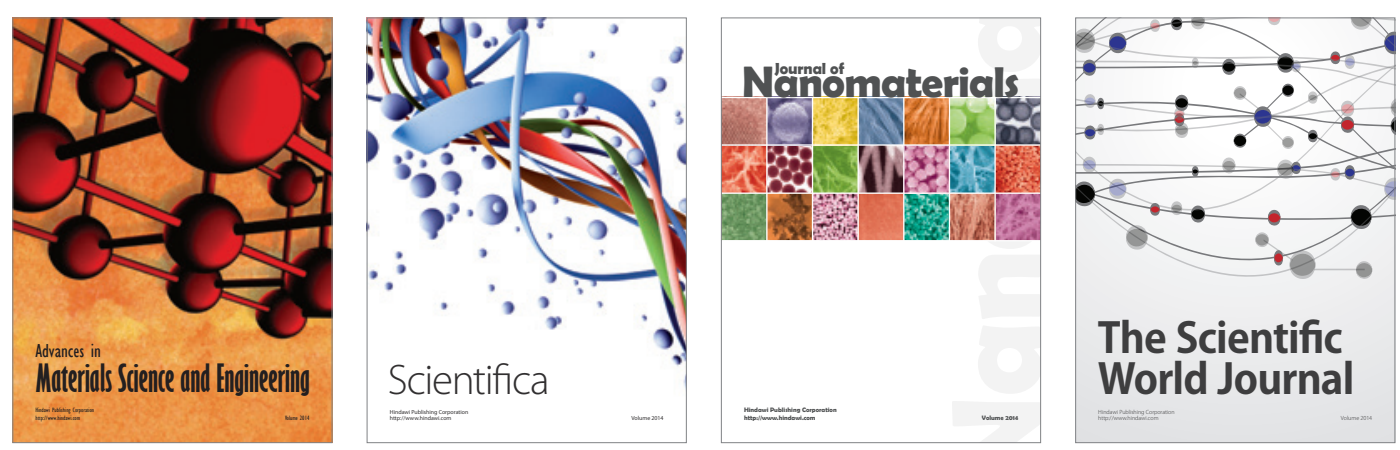

\section{The Scientific World Journal}
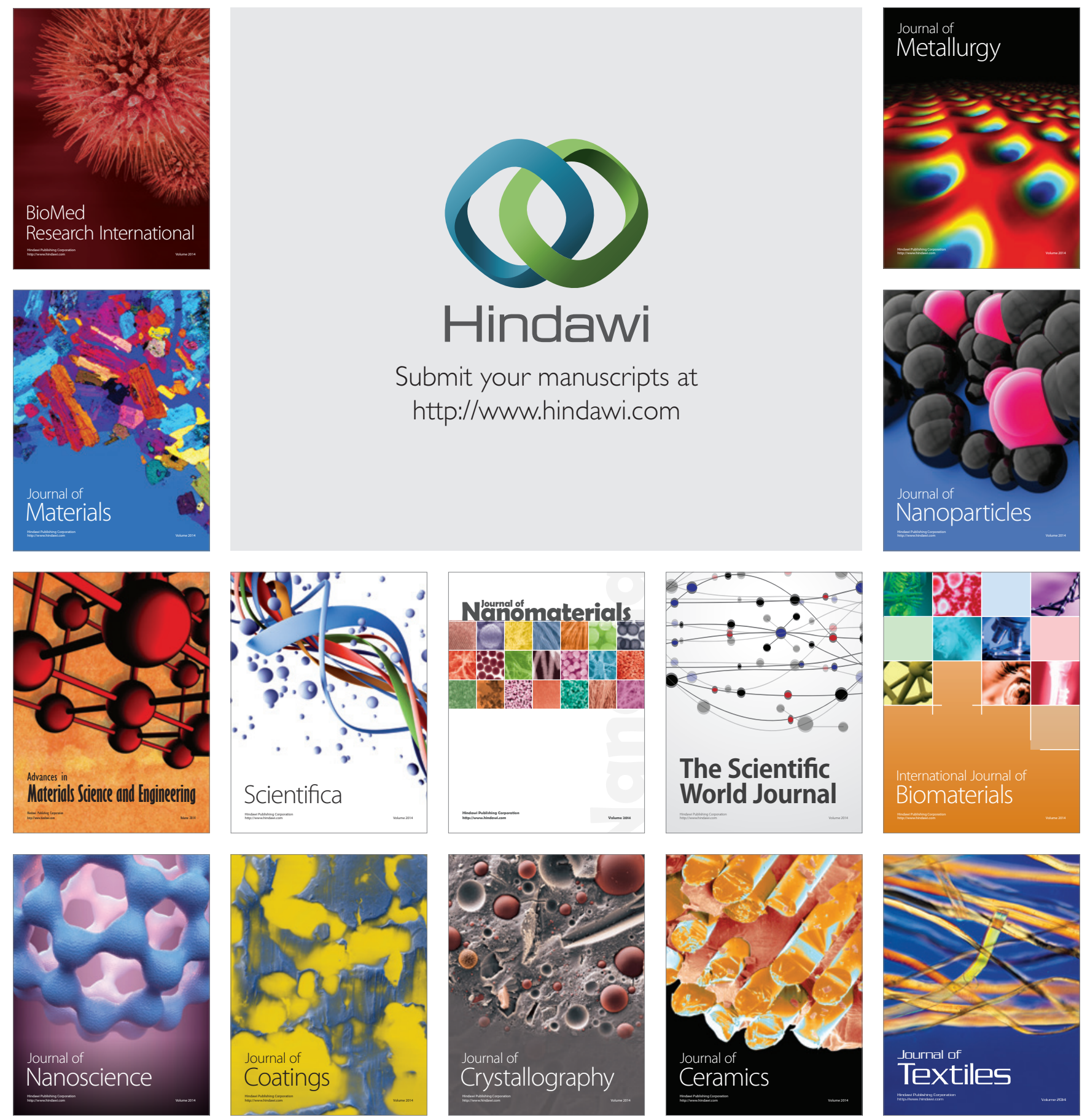